\title{
La lecture des ondes de coup de bélier sur le tracé de Bergeron,
}

\section{Reading of water hammer waves on a Bergeron diagram}

\author{
PAR A. SCHLAG \\ PROFESSEUR A L'UNIVERSITÉ DE TALGE, \\ DHECTEUR DU LABORATOHRE D'HYDTAULIOUE GÉNÉRAIF
}

\begin{abstract}
Dans une conduite soumise au phénomène du coup de bélier, l'épure de Bergeron permet de déterminer les «états» pression-débit, en autant de sections de la conduite et en autant d'instants qu'on le désire. Elle permet aussi de connaitre les ondes d'Alliévi $F$ et $f$ exprimant les variations conjointes de débit et de pression depuis l'origine du phénomène.
\end{abstract}

En fait, le phénomène du coup de bélier consiste en une succession d'ondes qui parcourent la conduite, en se croisant et se réfléchissant plus ou moins complètement aux points singuliers : extrémités, changements de section, nxuds... Plutôt qu'à des ondes se superposant à l'état initial, on a affaire $\dot{a}$ des ondes, $\dot{a}$ front plus ou moins raide, qui modifient l'état existant immédiatement avant leur passage.

Il est montré que ces ondes peuvent être lues sur l'épure de Bergeron. Leur considération conduit à l'établissement d'un lableau très représentatif du phénomène; elle permel aussi le calcul, sonvent aisé, de tous les états successif's dans les'diverses sections de la conduite.

La solution des problèmes de coup de bélier dans les conduites est basée sur les équations classiques d'Allievi :

$$
\mathrm{H}-\mathrm{H}_{0}=\mathrm{F}\left(t-\frac{x}{a}\right)+i\left(t+\frac{x}{a}\right)
$$

el :

$$
\frac{a}{g}\left(c_{0}-c\right)=\mathrm{F}\left(t-\frac{x}{a}\right)-f\left(t+\frac{x}{a}\right)
$$

Dans ces équations, $F$ et $f$ sont deux ondes de pression qui parcourent la conduite avec des vitesses égales à $\pm a$, et $(g / a) \mathrm{F}$ et $(g / a) /$, deux ondes de vitesse qui accompagnent ces ondes de pression.

Dans son remarquable ouvrage : $D u$ coup de bélier en hydraulique au coup de foudre en

\begin{abstract}
In a pipe subjected to the phenomenon of water hammer, the Bergeron diagram allows the determination of the pressure-flow conditions in as many sections of the pipe and at any instant desired. One can also determine the Allievi wave $F$ and $f$, expressing the twin variations of flow and pressure from the time the phenomenon begins.

In fact, the phenomenon of water hammer consists of a succession of wanes which traverse the pipe, passing and reflecting each other more or less entirely, between given points i.e. the ends of the pipe, changes in section, branches, etc.

Rather than waves superimposing themselves from the start, we have a system of waves, more or less steep fronted, which modify the state existing immediately ahead.

It is shown that these waves maty be plotted on a Bergeron diagram. Their consideration leads to the establishment of a chart which truely represents the phenomenon and also makes the subsequent calculation of all the successive states in the parions sections of the pipe easy.
\end{abstract}

électricité, Louis BERGERON énonce comme suit les lois exprimées par les formules d'Allievi :

« $1^{\circ}$ En un temps et un lieu quelconques le long d'une conduite, la variation de pression depuis l'origine du phénomène est égale à la somme de deux ondes de pression $F$ et $f$ qui s'y croisent à cet instant;

« $2^{\circ}$ Simultanément, la différence $\mathrm{F}-f$ de ces deux ondes est égale à $a / g$ fois la variation de la vitesse à ce temps et en ce lieu depuis l'origine du phénomène. »

(C'est nous qui soulignons les mots « depuis l'origine du phénomène $\gg$.)

$$
\text { *** }
$$

La résolution direcle des équations d'Allievi est assez souvent longue et parfois difficile. 
Mais, Louis Bergenon a développé et mis au point une méthode graphique de résolution actuellement bien connue de tous les hydrauliciens.

Dans la préface à l'ouvrage de Bergeron, M. E. G. Barillon, membre de l'Institut, écrit : "De tout ce qui se lit sur le bergeron, le lecteur du présent volume se rendra compte; il peut être assuré que dans ces voyages à travers les conduites hydrauliques ou les réseaux électriques, dans lesquels Louis Bergeron le guide pas à pas et lui montre les moindres particularités, il apprendra beaucoup, et que s'il est déjà préparé par la lecture d'ouvrages traitant la question par des méthodes purement mathématiques. il aura le plaisir de reconnaître au passage des phénomènes qui, jusqu'alors, ne lui étaient pas apparus dans toute leur clarté. »

Le but de la présente note est de montrer un de ces aspects qui, à notre connaissance tout au moins, n'a pas encore été signalé.

\section{***}

On sait que, pour le tracé du bergeron, on remplace, dans l'équation (2) les vitesses $c$ par les débits $Q$. Les équations (1) et (2) deviennent ainsi :

et :

$$
\mathrm{H}-\mathrm{H}_{0}=\mathrm{F}\left(t-\frac{x}{a}\right)+f\left(t+\frac{x}{a}\right)
$$

$$
\frac{a}{g \mathrm{~S}}\left(\mathrm{Q}_{0}-\mathrm{Q}\right)=\mathrm{F}\left(t-\frac{x}{a}\right)-f\left(t+\frac{x}{a}\right)
$$

$\mathrm{S}$ étant la surface de la section transversale de la conduite.

Le tracé peut donner l' « état» (pression, débit) pour toutes les sections et à tous les inslants que l'on désire $\left(^{*}\right)$.

Comme l'a montré Bergeron (fig. 9 de son ouvrage), il permet aussi de connaitre la grandeur des deux ondes de pression en un lieu et en

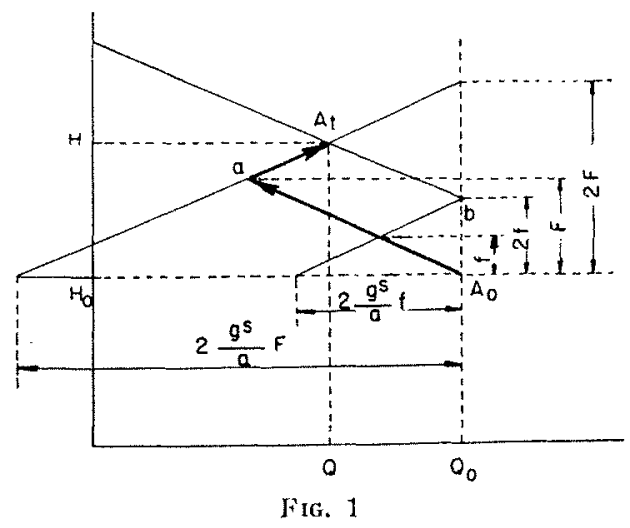

(*) Nous adoptons les notations $H$ et $Q$ pour les " itats 》 et $h$ et $q$ pour les pressinns et débits transportés par les ondes. un temps quelconques où le régime est connu. Si A.t est le point représentatif de l'état dans la section A à l'instant $t$, les ondes de pression, dans cette section, et à cet instant, sont représentées sur le bergeron, par la moitié des longueurs marquées $2 \mathrm{~F}$ et $2 f$ (fig. 1) $\left(^{* *}\right)$.

Remarquons qu'il s'agit bien, comme BERGERoN l'indique lui-même, d'ondes ayant provoqué une variation de pression depuis l'origine $d u$ phénomène.

Les ondes correspondantes de débit sont représentées par la moitié des longueurs :

$$
\text { 2. } \frac{g \mathrm{~S}}{a} \cdot \mathrm{F} \text { el } 2 . \frac{g \mathrm{~S}}{a} \cdot f
$$

Bien entendu, il s'agit encore d'ondes ayant provoqué une variation de débit depuis l'origine du phénomène.

$$
* *
$$

L'image du phénomène, déduite directement des équations d'Allievi, le représente donc comme dû à la superposition à l'état initial, des deux ondes $F$ et $f$ de pression, accompagnées des deux ondes de débit $(g \mathrm{~S} / a) \mathbf{F}$ et $(g \mathrm{~S} / a) f$.

Nous pensons qu'une représentation plus fidèle et plus parlante consisterait à considérer que chaque état dans une section quelconque résulte de l'état immédiatement précédent dans la même section, modifié par le passage, l'arrivée ou l'émission d'une ou plusieurs ondes conjointes de pression et de débit, circulant dans chaque tronçon à caractéristique unique.

Aux ondes $[\mathrm{F},(g \mathrm{~S} / a) \mathrm{F}]$ et $[f,(g \mathrm{~S} / a) f], s u$ perposées à l'état inifial, nous proposons de substituer, pour chaque intervalle de temps, une ou plusieurs ondes se superposant à l'état At pour donner l'état immédiatement suivant $\mathrm{A} t^{\prime}$

L'exposé de notre conception est plus simple dans le cas d'une perturbation de caractère instantané, entrainant dans les diverses sections de la conduite des variations brusques d'état. Nous nous placerons d'ahord dans cette hypothèse.

Nous aurons, par exemple, dans une section quelconque où la pression à l'origine était $\mathrm{H}_{0}$ la succession des pressions représentée figure 2.

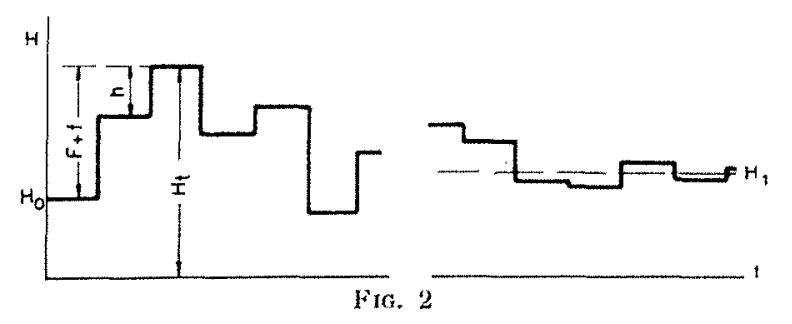

(**) Bergeron écrit (p. 15) que \& ce n'est là qu'une curiosité sans intérêt pour la résolution des problèmes qui se posent. 
Selon la conception classique, la pression $\mathrm{H}_{t}$ est égale à la pression à l'origine $\mathrm{H}_{0}$ augmentée de la somme algébrique $(\mathrm{F}+f)$ des pressions des ondes d'Allievi.

Nous proposons de considérer que la pression $\mathrm{H}_{t}$ résulte de la pression existant à l'instant précédent $\mathrm{H}_{t-1}$, augmentée de l'onde (ou de la somme de deux ondes) de pression $h$.

\section{2**}

En nous reportant à la figure 1 , nous voyons aisément que l'onde $[\mathrm{F},(g \mathrm{~S} / a) \mathrm{F}]$ peut être représentée par le vecteur AO.a et l'onde $[f,(g \mathrm{~S} / a) f]$ par le vecteur $a . \mathrm{A} t$.

Considérons maintenant deux états consécutifs dans une même section (fig. 3) At et A $t^{\prime}$.

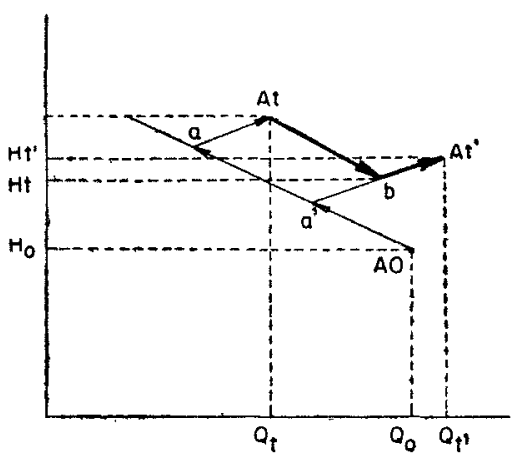

FIG. 3

Les ondes d'Allievi (depuis le début du phénomène) sont représentées respectivement par les tracés vectoriels $\mathrm{AO} . a$. At et $\mathrm{AO} . a^{\prime}$. A $t^{\prime}$.

Pour passer de l'état At à l'état immédiatement suivant $\mathrm{A} t^{\prime}$, on voit qu'on peut suivre le tracé vectoriel dessiné en trait fort $\mathrm{A} t . b . \mathrm{A} t^{\prime}$.

Les vecteurs At. $b$ et $b$. A $t^{\prime}$ représentent précisément respectivement les ondes incidente et réfléchie que nous proposons de considérer. Ces vecteurs sont souvent dessinés à priori sur le bergeron.

Ces ondes seront évidemment soumises, en ce qui concerne le coefficient angulaire des vecteurs qui les représentent, à la même règle de signe que les ondes $[\mathrm{F},(g \mathrm{~S} / a) \mathrm{F}]$ et $[f,(g \mathrm{~S} / a) f]$ : coefficient angulaire négatif pour l'onde $[\mathbf{F},(g \mathrm{~S} / a) \mathbf{F}]$ qui remonte la conduite et coefficient angulaire positif pour l'onde $[f,(g \mathrm{~S} / a) f]$ qui descend la conduite.

$\mathrm{Ce}$ coefficient angulaire est $\pm g \mathrm{~S} / a$. Posons, pour la facilité de l'écriture, $g \mathrm{~S} / a=\alpha$.

Nous écrirons donc :

pour une onde remontant la conduite:

$$
h=-\alpha q
$$

pour une onde descendant la conduite :

$$
h=+\alpha q
$$

Si les points $\mathrm{A} t$ et $\mathrm{A} t^{\prime}$ s'alignaient sur une direction + ou $-\alpha$, cela signifierait qu'une seule onde serait à envisager.

$$
\text { *** }
$$

Pour mieux illustrer notre conception, nous avons traité le début du phénomène du coup de bélier dans le cas simple suivant :

Conduite constituée de deux tronçons $\mathrm{AB}$ et $\mathrm{BC}$, longs respectivement de 100 et $180 \mathrm{~m}$, de sections 0,1 et $0,2 \mathrm{~m}^{2}$ et possédant des célérités respectives de 1.000 et $900 \mathrm{~m} / \mathrm{s}$. Si le tronçon $A B$ est ainsi parcouru par l'onde en un temps $l_{1} / a_{1}$

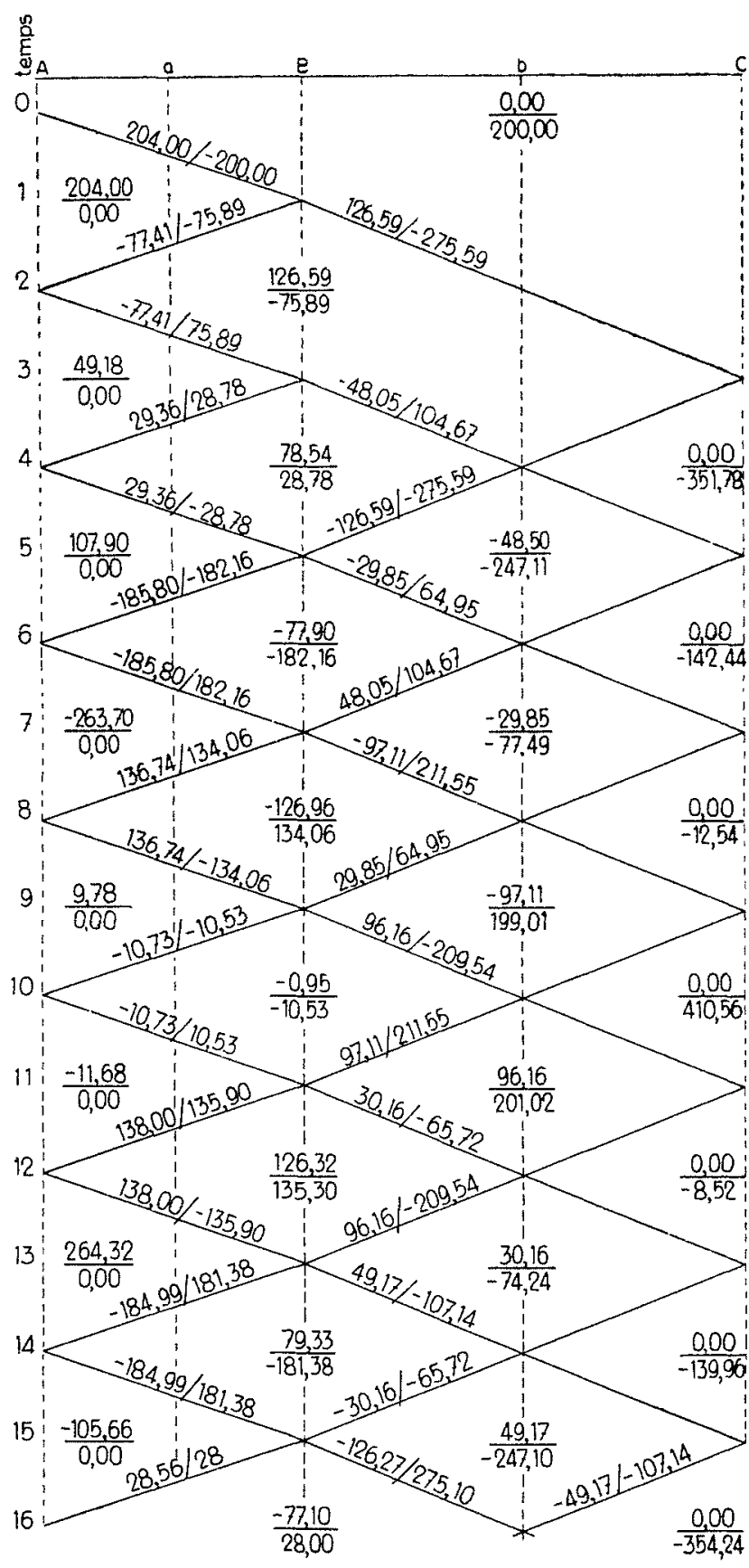

FIG. 4 
que nous prenons comme unité de temps, le tronçon BC sera parcouru en un temps égal à 2 unités.

Conditions initiales : pression $\mathrm{H}_{0}=200 \mathrm{~m}$; débit $Q_{0}=2001 / \mathrm{s}$.

Coup de bélier créé par la fermeture complète et instantanée de l'extrémité C. On ne tient pas compte du frottement.

Les divers états ont été relevés sur le bergeron et reportés à la figure 4 sous la forme conventionnelle $\mathrm{H} / \mathrm{Q}\left(^{\star}\right)$. On constatera que, ainsi que cela est connu, en un point quelconque, ces états varient d'une façon discontinue.

Les divers états sont séparés par des droites obliques qui représentent les ondes que nous proposons d'envisager et qui circulent à chaque instant dans la conduite; nous avons indiqué leurs valeurs. Il ne s'agit donc pas des ondes $[\mathrm{F},(g \mathrm{~S} / a) \mathbf{F}]$ et $[f,(g \mathrm{~S} / a) f])$ exprimées depuis l'origine du phénomène. Si noụs désignons par $\mathrm{H}^{\prime} / \mathrm{Q}^{\prime}$ et $\mathrm{H}^{\prime \prime} / \mathrm{Q}^{\prime \prime}$ deux états consécutifs dans une mème section, ce sont ces ondes qui font passer $\mathrm{H}^{\prime}$ à $\mathrm{H}^{\prime \prime}$ et $\mathrm{Q}^{\prime}$ à $\mathrm{Q}^{\prime \prime}$.

Par exemple, l'état de la section $b$, au milieu de $\mathrm{BC}$, avant le temps 4 , est :

$$
126,59 /-75,89 ;
$$

au temps 4 , il y arrive deux ondes :

$$
-48,05 / 104,67 \text { et }-126,59 /-275,89 \text {. }
$$

Par conséquent, l'état du point $b$ après 4 devient :

$$
\begin{gathered}
\mathrm{H}=126,59-48,05-126,59=-48,05 \\
\mathrm{Q}=-75,89+104,67-275,89=-247,11 \\
* *
\end{gathered}
$$

De cette conception physique du phénomène du coup de bélier, on peut déduire une méthode de calcul extrêmement simple et dont le mécanisme est, pensons-nous, très intuitif.

\section{Cas général}

Supposons qu'à une extrémité d'un tronçon à caractéristique unique, existe obligatoirement une loi $\varphi(\mathrm{Q}, \mathrm{H}, t)=0$ reliant le débit et la pression et qu'il y arrive une onde incidente $h_{i} / q_{i}$. Cette onde se réfléchit totalement ou partiellement en une onde réfléchie $h_{r} / q_{r}$.

Pour l'onde incidente :

$$
h_{i}= \pm \alpha q_{i}
$$

(*) $\mathrm{H}$ exprimé en mètres et $Q$ en $1 / \mathrm{s}$. et pour l'onde réfléchie :

$$
h_{r}=\mp \alpha q_{r}
$$

puisque ces deux ondes circulent en sens inverses.

L'état, qui était $\mathrm{Q}_{t} / \mathrm{H}_{t}$ avant l'arrivée de l'onde $i$ et sa réflexion, devient après :

$$
\mathrm{Q}_{t+1} / \mathrm{H}_{t+1} \text {. }
$$

L'état $(t+1)$ doit satisfaire aux équations suivantes :

$$
\begin{gathered}
\varphi\left(\mathrm{H}_{t+1}, \mathrm{H}_{t+1}, t+1\right)=0 \\
\mathrm{H}_{t+1}=\mathrm{H}_{t}+h_{i}+h_{r}=\mathrm{H}_{t} \pm \alpha\left(q_{i}-q_{r}\right) \\
\mathbf{Q}_{t+1}=\mathrm{Q}_{t}+q_{i}+q_{r}
\end{gathered}
$$

Si $\mathrm{H}_{t}, \mathrm{Q}_{t}, h_{i}$ et $q_{i}$ sont connus, les équations (3), (4) et (5) permettent de déterminer $q_{r}, \mathbf{Q}_{t+1}$ et (2) donne ensuite $h_{r}$.

\section{Examen de quelques cas particuliers}

\section{A) Le débit $Q$ est constant à l'extrêmité envisagée : $\mathrm{Q}=\mathrm{Q}_{0}$.}

Supposons qu'il arrive une onde incidente $h_{i} / q_{i}$ et que naisse une onde réfléchie : $h_{r} / q_{r}$ :

$$
h_{i}= \pm \alpha q_{i} \quad \mathrm{H}_{r}=\mp \alpha q_{\text {. }}
$$

Les équations (3), (4) et (5) deviennent :

$$
\begin{aligned}
& \mathbf{Q}_{t+1}=\mathbf{Q}_{0} \\
& \mathrm{H}_{t+1}=\mathrm{H}_{t} \pm \alpha\left(q_{i}-q_{r}\right) \\
& 0=q_{i}+q_{r}
\end{aligned}
$$

La résolution donne :

$$
\begin{aligned}
& q_{r}=-q_{i} \\
& h_{i}=h_{r} \\
& \mathrm{H}_{t+1}=\mathrm{H}_{t}+2 h_{i}
\end{aligned}
$$

\section{B) La pression est constante à l'extrémité envisagée : $\mathrm{H}=\mathrm{H}_{0}$.}

Les équations (3), (4) et (5) s'écrivent :

$$
\begin{aligned}
& \mathbf{H}_{t+1}=\mathbf{H}_{0} \\
& 0=\alpha\left(q_{i}-q_{r}\right) \\
& \mathbf{Q}_{t+1}=\mathbf{Q}_{t}+q_{i}+q_{r}
\end{aligned}
$$

La résolution donne:

d'où : $\quad q_{i}=q_{r}$

$h_{i}=-h_{r}$

$$
\mathrm{Q}_{t+1}=\mathrm{Q}_{t}+2 q_{i}
$$




\section{C) $Q$ passe brusquement de $Q_{t} \grave{a} Q_{t+1}$.}

Le problème se pose un peu différemment. On connaît $\mathrm{H}_{t}, \mathrm{Q}_{t}$ et $\mathrm{Q}_{t+1}$. Il n'y a pas d'onde incidente, mais il y' a émission d'une onde, que nous continuerons conventionnellement à appeler « réfléchie » $h_{r} / q_{r}$ avec $h_{r}= \pm \alpha q_{r}$.

Nous pourrons écrire :

et :

$$
\mathrm{Q}_{t+1}=\mathrm{Q}_{t}+q_{r}
$$

$$
\mathrm{H}_{t+1}=\mathrm{H}_{t}+h_{r}=\mathrm{H}_{t}=\alpha \cdot q_{r}
$$

Nous en déduisons :

$$
\begin{aligned}
& q_{r}=\mathrm{Q}_{\mathrm{t}+\mathrm{t}}-\mathrm{Q}_{t} \\
& h_{r}= \pm \alpha q_{r} \\
& \mathrm{H}_{t+1}=\mathrm{H}_{t}+h_{r} .
\end{aligned}
$$

\section{D) L'extrémité du tronçon envisagée est l'origine d'un autre tronçon.}

En $\mathrm{B}$, arrive une onde incidente :

$$
h_{\mathrm{i}} / q_{i} \text { avec } h_{i}= \pm \alpha q_{i}
$$

Elle est partiellement réfléchie dans ce troncon, en une onde $h_{r} / q_{r}$ avec $h_{r}=\mp \alpha q_{r}$, et une onde $h_{r}^{\prime} / q_{r}^{\prime}$ est émise dans le deuxième tronçon dans la même direction que l'onde $h_{i} / q_{i}$ :

$$
h_{r}^{\prime}= \pm \alpha^{\prime} q^{\prime}{ }_{r}
$$

Avant l'arrivée de l'onde, il y avait mêmes pressions $\mathrm{H}_{t}$ et mêmes débits $\mathrm{Q}_{t}$ de part et d'autre de la section $B$. Après cette arrivée, il y a encore égalité des pressions $\mathrm{H}_{t+1}$ et égalité des débits $Q_{t+1}$.

Dans le tronçon par où arrive l'onde $h_{i} / q_{i}$ :

$$
\begin{aligned}
& \mathrm{H}_{t+1}=\mathrm{H}_{t}+h_{i}+h_{r} \\
& \mathrm{Q}_{t+1}=\mathrm{Q}_{t}+q_{i}+q_{r}
\end{aligned}
$$

Dans l'autre tronçon :

$$
\begin{aligned}
& \mathrm{H}_{t+1}=\mathrm{H}_{t}+h^{\prime}{ }_{r} \\
& \mathrm{Q}_{t+1}=\mathrm{Q}_{t}+q^{\prime}{ }_{r}
\end{aligned}
$$

La résolution de ces équations donne:

$$
\begin{gathered}
q_{r}=\frac{\alpha-\alpha^{\prime}}{\alpha+\alpha^{\prime}} q_{i} \\
q_{r}^{\prime}=\frac{2 \alpha}{\alpha+\alpha^{\prime}} q_{i}\left(^{*}\right)
\end{gathered}
$$

$\left.{ }^{\star}{ }^{\star}\right)$ Ces formules sont analogues à celles que l'on rencontre dans ia théoric du passage d'une onde lumineuse plane d'un milieu d'indice $t_{2}$ dans un autre d'indice $t_{2}$, lorsque le plan d'onde est parallèle au plan de séparation des deux milienx. Entre les amplitudes de ces ondes, on a les relations :

$$
a_{2}=\frac{2 \alpha}{\alpha+\alpha^{\prime}} a_{i} \quad a_{r}^{\prime}=\frac{\alpha-\alpha^{\prime}}{a+\alpha^{\prime}} a_{i}
$$

avec $u=c / v$ : $a^{\prime}=c / v^{\prime}$

e:vitesse de la lumière dans le vide, $b, v^{\prime}$ : vitesses dans les milicux considérés.

\section{E) Cas d'une bifurcation simple.}

Dans un des tronçons de la bifurcation arrive une onde $h_{i} / q_{i}$. Une onde $h_{r} / q_{r}$ est réfléchie dans ce tronçon et des ondes $h^{\prime}{ }_{r} / q^{\prime}{ }_{r}$ et $h^{\prime \prime}{ }_{r} / q^{\prime \prime}{ }_{r}$ sont émises dans les deux autres tronçons.

Compte tenu du sens de circulation des ondes dans les divers troncons, on a :

$$
\begin{aligned}
& h_{i}= \pm \alpha q_{i} \\
& h_{r}=\mp \alpha q_{r} \\
& h^{\prime}{ }_{r}= \pm \alpha^{\prime} q^{\prime}{ }_{r} \\
& h^{\prime \prime}{ }_{r}= \pm \alpha^{\prime \prime} q^{\prime \prime}{ }_{r} .
\end{aligned}
$$

Au noud, à chaque instant $\mathrm{H}$ est le même quel que soit le tronçon que l'on considère; de plus, $\mathrm{Q}=\mathrm{Q}^{\prime}+\mathrm{Q}^{\prime \prime}$.

Nous aurons donc à l'instant $t+1$ :

$$
\mathrm{H}_{t+1}=\mathrm{H}_{t}+h_{i}+h_{r}=\mathrm{H}_{t}+h_{r}^{\prime}=\mathrm{H}_{t}+h^{\prime \prime}{ }_{r}
$$
et :

$$
\frac{\mathrm{Q}_{t}+q_{i}+q_{r}}{\mathrm{Q}_{t+1}}=\frac{\mathrm{Q}_{t}+q^{\prime}{ }_{r}}{\mathrm{Q}_{t+1}^{\prime}}+\frac{\mathrm{Q}^{\prime \prime}{ }_{t}+q^{\prime \prime}{ }_{r}}{\mathrm{Q}^{\prime \prime}{ }_{t+1}}
$$

soit :

$$
\begin{aligned}
& h_{i}+h_{r}=h_{r}^{\prime} \quad \text { ou } \quad \alpha q_{i} \cdots \alpha q_{r}=\alpha^{\prime} q^{\prime}{ }_{r} \\
& h^{\prime}=h^{\prime \prime}{ }_{r} \quad \alpha^{\prime} q^{\prime}{ }_{r}=\alpha^{\prime \prime} q^{\prime \prime}{ }^{\prime} \\
& q_{i}+q_{r}=q_{r}^{\prime}+q^{\prime \prime}{ }_{r}
\end{aligned}
$$

soient trois équations qui permettent de trouver Jes trois inconnues:

$$
\begin{aligned}
q_{r}^{\prime} & =\frac{2 \alpha \alpha^{\prime \prime}}{\alpha \alpha^{\prime}+\alpha^{\prime} \alpha^{\prime \prime}+\alpha^{\prime \prime \alpha} \alpha} q_{i} \\
q^{\prime \prime}{ }_{r} & =\frac{2 \alpha \alpha^{\prime}}{\alpha \alpha^{\prime}+\alpha^{\prime} \alpha^{\prime \prime}+\alpha^{\prime \prime} \alpha} q_{i} \\
q_{r} & =\frac{\alpha \alpha^{\prime}-\alpha^{\prime} \alpha^{\prime \prime}+\dot{\alpha}^{\prime \prime} \alpha}{\alpha \alpha^{\prime}+\alpha^{\prime} \alpha^{\prime \prime}+\alpha^{\prime \prime} \alpha} q_{i}
\end{aligned}
$$

On en déduit facilement $h_{r}^{\prime}, h^{\prime \prime}, h_{r}, \mathbf{H}_{i+1}$ et $\mathrm{Q}_{t+1}$

Si les temps de parcours des ondes dans les divers tronçons avaient une commune mesure, deux ou trois ondes pourraient arriver en même temps au nœud; il suffirait alors de considérer séparément chacune des ondes incidentes et de faire la somme, pour chaque tronçon, de toutes les ondes réfléchies on émises.

\section{F) Cas d'une manouvre progressive.}

Nous avons jusqu'ici, dans les exemples que nous avons traités, supposé que la manœuvre 
génératrice du coup de bélier étail instantanée.

Si elle était progressive, il faudrait la considérer comme constituće d'une succession de pelites manouvres instantanées. Il y aurait à chaque instant émission, circulation, réflexion, etc. de petites ondes, remplaçant les modifications continues réelles de pression et de débit.

\section{Conclusion}

La conception du phénomène du coup de bélier que nous avons exposée ici résulte directement du tracé de Bergeron. Nous pensons que tout en n'en étant pas différente en principe, elle correspond de plus près à la réalité physique que celle où l'on fait intervenir des ondes ayant provoqué des variations de pression et de débit « depuis l'origine du phénomène ».

Nous en avons déduit :

- Un tableau de représentation très intuitif du phénomène (fig. 4);

- Une méthode de calcul, souvent simple et aussi exacte qu'on le désire, des « états » successifs d'autant de sections que l'on veul, ainsi que des « ondes » qui circulent dans la conduite ou le réseau.

Nous ne prétendrons pas que cette méthode soit supérieure à celle imaginée par BERGERoN. Cielle-ci possède des avantages bien connus : simplicité et automatisme d'application qui permettent d'en confier l'exécution à un dessinateur soigneux, facilité également de repérer une erreur éventuelle, etc.

Nous pensons cependant que notre méthode n'est pas dépourvue d'intérêt. Elle pourrait même parfois réclamer à son tour, en sa faveur, certains avantages, par exemple, si l'on désirait considérer un amortissement de l'onde au cours de son trajet. Il suffirait alors dans le calcul de tenir compte que l'onde émise à une extrémité d'un tronçon de conduite, arrive à l'autre extrémité, affectée du coefficient d'amortissement convenable. Il ne s'agit pas, bien entendu, d'une perte de pression par frottement hydraulique, mais d'un véritable amortissement tel que celui que subissent les ondes de choc se transmettant dans les corps solides.

\section{I S C U S I O N}

président : M. Remenienas

M. le Président remercie M. Schlaci et souligne l'intérêt didactique de sa nouvelle interprétation de la célèbre méthode graphique; il souhaite que les ingénieurs des bureaux d'études utilisent dans divers cas types la méthode de $M$. BERGeron suivant le procédé de M. Schlag et nous fassent connaître, à la lumière de leur expérience les avantages et inconvénients qu'elle présente en pratique par rapport aux modes d'emploi usuels.

M. le Président sait tout particulièrement gré à M. Schlag d'avoir adopté une suggestion faite en son temps par M. le Président Barrillon et suivant laquelle, en hommage à son éminent inventeur, on devraic appeler l'spure sui vous occupe un «bergeron ", tout comme en stafique graphique l'on dit un «cremona » et non une épure de Cremona.

Devant les remarques fort intéressantes de M. Schlaci qui l'ont conduit à un mode de calcul inédit, M. Paul BERGERon voudrait essayer de montrer sommairement les différences qui, à son avis, existent entre les moyens proposés par MM. Arurevi, Schlag et L. BerGERON pour la solution du même problème.

Considérons le cas simple d'une fermeture progressive de Ioi quelconque sur une conduite gravitaire. Raisonnons sur l'épure tracée par la méthode graphique qui permet de mettre en évidence chacune des trois interprètations :

$1^{\circ}$ En considérant les ondes depuis l'origine (interprétation Allievi), l'ćtat au point $t_{i: 2}$ résulte de la superposition à l'état origine $A_{0}\left(h_{*} q_{o}\right)$ de deux ondes qui se croisent, soit $F_{i+2}$ qui remonte le courant, et $f_{t+2}$ qui le descend. Chacune de ces ondes de pression est liée à une variation de débit telle que l'onde $f_{t+2}$ peut être représentée par le vecteur $A_{0} \mathrm{M}$ et l'onde $\mathrm{F}_{1+2}$ par le vecteur $\mathrm{M} \mathrm{A}_{i+2}$.

$2^{\circ}$ M. Scurag définit l'état en $A_{t, 2}$ comme résultant de la superposition à l'état existant non plus en $A_{0}$, mais en $A_{i}$, de deux ondes intermédiaires, I'une représentée par le vecteur $A_{6} B_{i+1}$ appelée par lui onde incidente, et l'autre représentée par le vecteur $B_{i}+I A_{i+2}$ appelée onde réfléchie.

Les ondes et les états qui ont précédé celui existant en $A_{i}$ ne sont plus à considérer.

$3^{\circ}$ La méthode graphique, dans sa réalisation pratique, ne fait plus appel à la notion des ondes qui se croisent et peut oublier tout le passé qui a précédé, dans l'exemple choisi, l'existence de l'état en $B_{i+1}$.

Get état en $B_{i+1}$ soit $h_{i}+I q_{t+1}$ constitue une onde qui se propage à la célérité « $a$ » en restant identique à elle-mênc; mais si les conditions rencontrées sur le parcours imposent une modification de l'état $h_{1}+I q_{i+1}$, l'application $\mathrm{du}$ théorème des quantités de mouvement montre que $\Delta h= \pm a / g s \Delta q$, c'est-à-dire que le nouvel etat doit être obligatoirement sur la droite qui passe par $B_{i+1}$ et de coefficient angulaire $\pm a / g s$. Dans ces conditions, à l'arrivée au point $A$, l'état $A_{i, 2}$ sera situć sur cette droite, et devant satisfaire d'autre part à la loi $f(q h t)=0$ existant en $A_{\imath+2}$ au temps $i+2$, sera à l'intersection de la droite et de la courbe donnant $h=f(q)$ au temps $i+2$.

Faussée peut-être par la méthode graphique, cette conception, basée sur la simple considération de l'onde émise par l'état existant en un point el en un temps donnés, dont les conditions de variation s'appuient sur l'application élémentaire du théorème des quantités de 
(2) 